\title{
How much quantum noise of amplifiers is detrimental to entanglement
}

\author{
G. S. Agarwal ${ }^{\mathrm{a}}$, S. Chaturvedi ${ }^{\mathrm{b}}$ \\ ${ }^{a}$ Department of Physics, Oklahoma State University, Stillwater, OK 74078, USA \\ ${ }^{b}$ School of Physics, University of Hyderabad, Hyderabad 500046, India
}

\begin{abstract}
We analyze the effect of the quantum noise of an amplifier on the entanglement properties of an input state. We consider both phase insensitive and phase sensitive amplification and specialize to Gaussian states for which entanglement measures are well developed. In the case of phase insensitive amplification in which both the modes are symmetrically amplified, we find that the entanglement in the output state vanishes if the intensity gain exceeds a limiting value $2 /\left(1+\exp \left[-E_{N}\right]\right)$ where $E_{N}$ is the logarithmic negativity of the input state which quantifies the initial entanglement between the two modes. The entanglement between the two modes at the output is found to be more robust if only one mode is amplified.
\end{abstract}

Modifications of quantum features of light propagating through absorbing and amplifying media has been a subject of considerable importance and hence of intense activity [1]-11]. For obvious practical reasons, the process of amplification has been of special interest and for the case of a single mode, using specific mathematical models for amplification, various authors have derived limits on the amplifier gain beyond which the noise, intrinsic to the process of amplification, destroys special non classical features such as squeezing and subPoissonian statistics. Similar limits, for the case when the output ceases to have any non classical properties at all, have also been obtained. Motivated by these studies, our aim here is to answer similar questions in the context of quantum entanglement which has now come to be universally recognized as an indispensable resource for quantum information processing. To be specific, we consider the effect of amplification on the two mode squeezed vacuum state which falls in the category of much studied entangled Gaussian states which occupy a privileged position in quantum information processing through continuous variable systems [12]. Not only have they been put to use in experimental demonstrations of teleportation [13] and quantum cryptography, they have also been found to be amenable to resolution of questions concerning their separability [14] -[16]

Email addresses: girish.agarwal@okstate.edu (G. S. Agarwal), scsp@uohyd.ernet.in (S. Chaturvedi)

Preprint submitted to Elsevier

June 1, 2018 
and distillability [17].

In this letter we consider two kinds of amplifiers- phase insensitive and phase sensitive. The two amplification processes are quite distinct in character in so far as their entanglement properties are concerned. While the phase insensitive amplifier performs local operations on the two modes, albeit non unitary, phase sensitive amplification operates quite differently. Our key results on the critical values of the gain are given by eqs. (19) and (23). For phase sensitive amplifier we give a condition on the phase mismatch that can be tolerated before the entanglement in the output starts deteriorating. Interestingly enough, Josse et al [10] have demonstrated a linear optical implementation of optical amplification process and some of the ideas of this paper can be tested by methods of linear optics. We consider phase insensitive amplification first.

I. Phase Insensitive Amplifier: We model this type of amplifier in the standard way as a bath consisting of $N$ two level atoms of which $N_{1}$ are in the excited state and $N_{2}$ in the ground state with $N_{1}>N_{2}$. Under the assumptions that atomic transitions have a large width and that the bath is maintained in a steady state, the time evolution of the density operator $\rho$ for a single mode of radiation field on resonance with the atomic transition is described, in the interaction picture, by the master equation

$$
\begin{aligned}
\frac{\partial \rho}{\partial t}= & -\kappa N_{1}\left(a a^{\dagger} \rho-2 a^{\dagger} \rho a+\rho a a^{\dagger}\right) \\
& -\kappa N_{2}\left(a^{\dagger} a \rho-2 a \rho a^{\dagger}+\rho a^{\dagger} a\right)
\end{aligned}
$$

where $a$ and $a^{\dagger}$ are the annihilation and creation operators of the field mode. An optical realization of (11) for $N_{2}=0$ is discussed in [10]. An important feature of this master equation is that if the initial density operator is a Gaussian then its Gaussian character is preserved at later times. In particular, an initial Gaussian Wigner distribution evolves into a Gaussian Wigner distribution. The celebrated result of Hong Friberg and Mandel [3] states that both subPoissonian statistics as well as the squeezing characteristics of any input state (not necessarily Gaussian) survive if the gain $|G|^{2}$ where $G \equiv \exp \left[\kappa t\left(N_{1}-N_{2}\right)\right]$ of the amplifier satisfies the condition

$$
|G|^{2}<\frac{2 N_{1}}{N_{1}+N_{2}}<2 .
$$

The evolution of the density operator under the master equation above is entirely captured by the following intuitively appealing Heisenberg like evolutions for the mode creation and annihilation operators:

$$
a(t)=G a(0)+c^{\dagger}, a^{\dagger}(t)=G^{*} a^{\dagger}(0)+c^{\dagger},
$$

where the averages of the noise operators $c c^{\dagger}$ and $c^{\dagger} c$ are taken to be

$$
<c c^{\dagger}>=(1+\eta)\left(|G|^{2}-1\right), \quad\left\langle c^{\dagger} c>=\eta\left(\left|G^{2}\right|-1\right),\right.
$$

with $\eta=N_{2} /\left(N_{1}-N_{2}\right)$. Hereafter we would take (3) and (4), supplemented by the conditions $|G|^{2}>1$ and $\eta \geq 0$ as the equations defining the action of a linear 
phase insensitive quantum amplifier. Alternatively, one may view these equations as arising entirely from the requirements of preservation of commutation relations supplemented by reasonable phenomenological inputs. The condition that $\left[a(t), a^{\dagger}(t)\right]=1$, given $\left[a(0), a^{\dagger}(0)\right]=1$, requires that $\left[c, c^{\dagger}\right]=|G|^{2}-1$ and hence $\left\langle c c^{\dagger}\right\rangle-\left\langle c^{\dagger} c\right\rangle=|G|^{2}-1$ and the noise correlations as in (4) are consistent with this equality. This reformulation of the master equation above not only facilitates computations but also relates directly to experimentally relevant quantities. It should be kept in mind that the noise source is Gaussian in nature.

For continuous variable systems, entanglement measures are well developed for Gaussian states and thus for our quantitative studies we have chosen to examine the effects of quantum noise of the amplifier on Gaussian entangled states.

In order to study the evolution of quantum entanglement of a Gaussian state $\rho$ as it is amplified, in a manner as prescribed above, we make use of the well known criteria for entanglement in Gaussian states developed in [14]-[16] where the covariance matrix $\sigma$ of the Wigner distribution,

$$
\begin{array}{r}
W(X)=\frac{e^{-(X-<\hat{X}>) \sigma^{-1}(X-<\hat{X}>)^{T} / 2}}{(2 \pi)^{n} \sqrt{\operatorname{Det}(\sigma)}}, \\
X \equiv\left(x_{1}, p_{1}, \cdots, x_{n}, p_{n}\right),
\end{array}
$$

associated with the $n$ mode Gaussian state $\rho$ plays a key role. The elements of covariance matrix $\sigma$, are given by

$$
\sigma_{i j}=\frac{1}{2}<\left(\hat{X}_{i} \hat{X}_{j}+\hat{X}_{j} \hat{X}_{i}\right)>-<\hat{X}_{i}><\hat{X}_{j}>
$$

where $\hat{X} \equiv\left(\hat{x}_{1}, \hat{p}_{1} \cdots, \hat{x}_{n}, \hat{p}_{n}\right), \hat{x}_{j}=\left(a_{j}+a_{j}^{\dagger}\right) / \sqrt{2}, \hat{p}_{j}=\left(a_{j}-a_{j}^{\dagger}\right) / \sqrt{2} i, a_{j}$ $\left(a_{j}^{\dagger}\right)$ denote the bosonic annihilation (creation) operators associated with the $j^{\text {th }}$ mode, and $<\cdot>\equiv \operatorname{Tr}[\cdot \rho]$. By definition, the covariance matrix is a real positive symmetric matrix and, by Williamson's theorem, is therefore congruent by a symplectic transformation to a diagonal matrix. The entries along the diagonal, the symplectic eigenvalues, can then be used to constrain and characterize the covariance matrices associated with a quantum state [18]. The idea that violations of these constraints by the symplectic eigenvalues of the 'partially transposed' density operator $\tilde{\rho}$ corresponding to the Gaussian state $\rho$ can be viewed as a signal for entanglement, has been ingeniously put to use in [14] -16 ] to arrive at the necessary and sufficient conditions for separability of bipartite Gaussian states.

In the two mode case, the covariance matrix has the form

$$
\sigma=\left(\begin{array}{cc}
\alpha & \gamma \\
\gamma^{T} & \beta
\end{array}\right)
$$

Given the covariance matrix for a two mode state $\rho$, the two symplectic eigenvalues of the covariance matrix associated with its 'partial transpose' $\tilde{\rho}$ are 
explicitly given by ,

$$
\tilde{\nu}_{ \pm}=\sqrt{\frac{\tilde{\Delta}(\sigma) \pm \sqrt{\tilde{\Delta}(\sigma)^{2}-4 \operatorname{Det}(\sigma)}}{2}}
$$

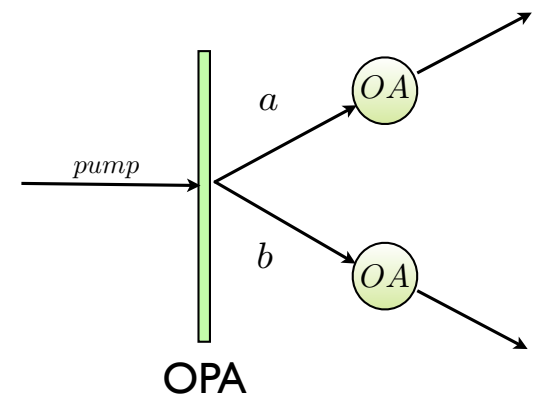

Figure 1: Schematic diagram for the amplification of a two mode entangled Gaussian state by a phase insensitive amplifier. The optical parametric amplifier (OPA) produces a two mode squeezed vacuum state of $a$ and $b$. In the symmetric case, both the optical amplifiers (OA) are present. In the asymmetric case the OA from the $b$ arm is removed.

where $\tilde{\Delta}(\sigma)=\operatorname{Det}(\alpha)+\operatorname{Det}(\beta)-2 \operatorname{Det}(\gamma)$. If $\tilde{\nu}_{<}$is taken to denote the smaller of the two symplectic eigenvalues, then the necessary and sufficient conditions for $\rho$ to be an entangled state can be expressed as

$$
\tilde{\nu}_{<}<\frac{1}{2} \text {. }
$$

A possible quantitative measure of entanglement, discussed in detail in [19, 20], may be taken to be the logarithmic negativity $E_{N}(\rho)$ :

$$
E_{N}(\rho)=\max \left[0,-\ln \left(2 \tilde{\nu}_{<}\right)\right] .
$$

For the special case when $\sigma$ has the form

$$
\sigma=\left(\begin{array}{cccc}
A & 0 & B & C \\
0 & A & C & -B \\
B & C & A^{\prime} & 0 \\
C & -B & 0 & A^{\prime}
\end{array}\right),
$$

the two symplectic eigenvalues $\tilde{\nu}_{ \pm}$turn out to be

$$
\tilde{\nu}_{ \pm} \equiv \frac{1}{2}\left[\left(A+A^{\prime}\right) \pm \sqrt{\left(A-A^{\prime}\right)^{2}+4\left(B^{2}+C^{2}\right)}\right] .
$$

These expressions will be useful later. For the two mode squeezed vacuum

$$
\begin{aligned}
& \rho=S(z)|0,0><0,0| S^{\dagger}(z), \\
& S(z)=\exp \left[z a^{\dagger} b^{\dagger}-z^{*} a b\right], z=r e^{i \theta},
\end{aligned}
$$


which belongs to the class of Gaussian entangled states, the two symplectic eignevalues are $\tilde{\nu}_{ \pm}=e^{ \pm 2 r} / 2$ and thus the quantum entanglement in in this state is directly linked to the squeezing parameter $r$ as the logarithmic negativity $E_{N}=2 r$. As $r \rightarrow 0, \tilde{\nu}_{<} \rightarrow 1 / 2$ and the entanglement disappears. Using (3) and (4) we next discuss the dynamics of entanglement and consider two cases. A The Symmetric Case Here we examine the situation when both the modes are symmetrically amplified as shown schematically in the Fig.1.

On making the replacements appropriate to this case

$$
a \longrightarrow G a+c^{\dagger}, b \longrightarrow G b+d^{\dagger},
$$

and using

$$
\begin{aligned}
& <c c^{\dagger}>=(1+\eta)\left(|G|^{2}-1\right),<c^{\dagger} c>=\eta\left(\left|G^{2}\right|-1\right), \\
& <d d^{\dagger}>=(1+\eta)\left(|G|^{2}-1\right),<d^{\dagger} d>=\eta\left(\left|G^{2}\right|-1\right),
\end{aligned}
$$

with all other noise averages set equal to zero, one finds that entries in (12) are given by

$$
\begin{aligned}
& A=A^{\prime}=\left[|G|^{2} \cosh 2 r+(1+2 \eta)\left(|G|^{2}-1\right)\right] / 2, \\
& B=\left[|G|^{2} \sinh 2 r \cos \theta\right] / 2, C=\left[|G|^{2} \sinh 2 r \sin \theta\right] / 2,
\end{aligned}
$$

and hence

$$
\tilde{\nu}_{<}=\left[|G|^{2}\left(e^{-2 r}+(1+2 \eta)\right)-(1+2 \eta)\right] / 2 .
$$

Requiring that the output state remain an entangled state i.e. $\tilde{\nu}_{<}<1 / 2$ then translates into the following condition for the gain :

$$
|G|^{2}<\left(\frac{2+2 \eta}{1+2 \eta+e^{-2 r}}\right) .
$$

In the special case of a fully inverted amplifier, $\eta \rightarrow 0$, and we have

$$
|G|^{2}<\frac{2}{\left(1+e^{-2 r}\right)}=\frac{2}{\left(1+e^{-E_{N}}\right)},
$$

where $E_{N}$ is the logarithmic negativity of the input state. Further, for maximum entanglement in the initial state i.e. $r \rightarrow \infty$, this becomes $|G|^{2}<2$. We note that Scheel et al. [21] using the concept of relative quantum entropy derived a result equivalent to (20). Hillery and Zubairy [22] used the high gain limit and concluded that in this limit all entanglement would be lost. These papers however do not use the logarithmic negativity criteria. It is interesting to note that conditions similar to (19) are found for the case of loss of entanglement [15] by attenuators which have been extensively studies [21, 23, 24].

$B$ The Asymmetric Case Next we consider the case when, say only the mode $a$ is amplified. On making the replacements

$$
a \longrightarrow G a+c^{\dagger}, b \longrightarrow b,
$$




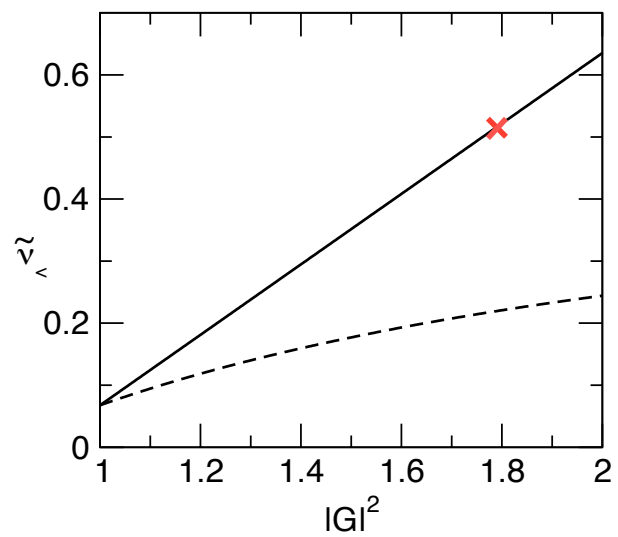

Figure 2: Variation of the entanglement measure $\tilde{\nu}_{<}$as a function of the gain $|G|^{2}$ for the symmetric case (solid line) and the asymmetric case (dashed line) for $r=1, \eta=0$. The cross marks the critical value of the gain in the symmetric case beyond which the entanglement in the output state vanishes.

and and using (15) we obtain

$$
\begin{aligned}
& A=\left[|G|^{2} \cosh 2 r+(1+2 \eta)\left(|G|^{2}-1\right)\right] / 2, \\
& A^{\prime}=[\cosh 2 r] / 2, B=[|G| \sinh 2 r \cos \theta] / 2, \\
& C=[|G| \sinh 2 r \sin \theta] / 2,
\end{aligned}
$$

which give

$$
\begin{aligned}
\tilde{\nu}_{<}= & \frac{1}{4}\left[\left(\left|G^{2}\right|+1\right) \cosh 2 r+(1+2 \eta)\left(|G|^{2}-1\right)\right. \\
& \left.-\sqrt{\left(|G|^{2}-1\right)^{2}(\cosh 2 r+1+2 \eta)^{2}+4|G|^{2} \sinh ^{2} 2 r}\right]
\end{aligned}
$$

In contrast to the symmetric case, here one finds $\tilde{\nu}_{<}$is always less than $1 / 2$ for $\eta=0$ and therefore the entanglement survives no matter how large the gain is. The variation of $\tilde{\nu}_{<}$in the two cases as a function of the gain is shown in the Fig. 2. The situation however is different if $\eta$ is non zero. In this case there is a threshold value of gain beyond which the entanglement of the initial state is lost. The threshold value of the gain depends on the value of $\eta$. Clearly for larger $\eta$ the entanglement degrades faster. We show this behavior in the Fig 3. As mentioned in the introduction, our results like (20) and (23) can be tested purely by means of linear optics [10]. II Phase Sensitive Amplification: We next consider a phase sensitive amplifier as shown schematically in the Fig. 4. The net amplification in this case depends on the relative phase $\left(\theta-\theta^{\prime}\right)$. Interesting interference effects in OPA cavities due to pumping with squeezed light were predicted and verified [25]-227]. 


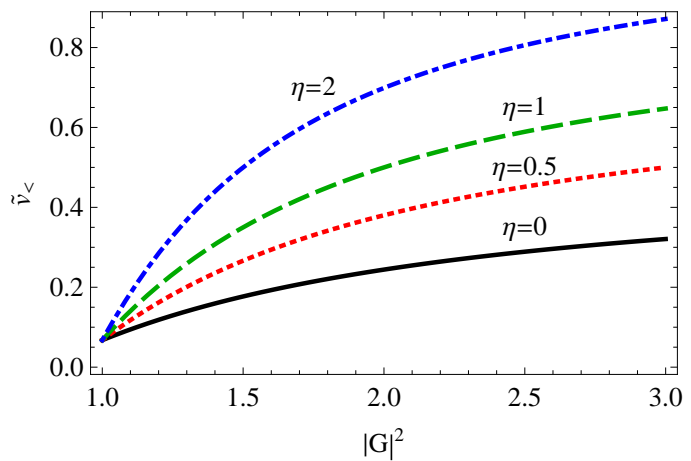

Figure 3: Variation of the entanglement measure $\tilde{\nu}_{<}$as a function of the gain $|G|^{2}$ for different $\eta$ and the squeezing parameter $r=1 . \eta=0$ (Solid line), $\eta=0.5$ (Dotted line), $\eta=1$ (Dashed line), $\eta=2$ (Dotdashed line).

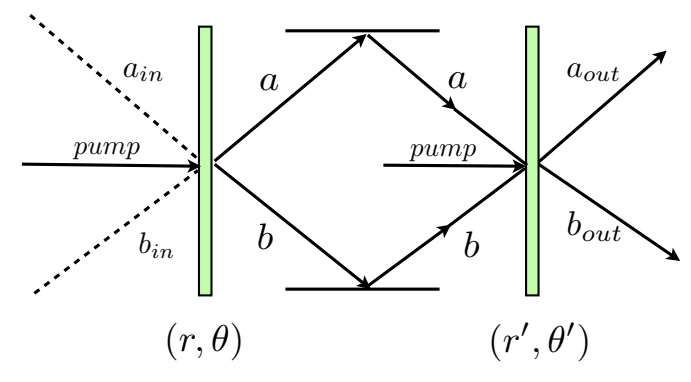

OPA OPA

Figure 4: Schematic diagram of a phase sensitive amplifier. The first OPA produces a squeezed vacuum state of the modes $(a, b)$ and the second OPA acts as an amplifier.

The action of this amplifier is to transform the input squeezed vacuum $S(z)|0,0><0,0| S^{\dagger}(z)$ to the state $S\left(z^{\prime}\right) S(z)|0,0><0,0| S^{\dagger}(z) S^{\dagger}\left(z^{\prime}\right)$. Using the composition theorem for squeezing operators [28],

$$
\begin{aligned}
& S\left(z_{1}\right) S\left(z_{2}\right)=S\left(z_{3}\right) e^{i\left(a^{\dagger} a+b^{\dagger} b+1 / 2\right) \Phi}, \quad z_{i}=r_{i} e^{i \theta_{i}}, \\
& \zeta_{3}=\frac{\zeta_{1}+\zeta_{2}}{1+\zeta_{1}^{*} \zeta_{2}}, \quad, \zeta_{i}=\tanh r_{i} e^{i \theta_{i}} \\
& \Phi=\frac{1}{2 i} \ln \left(\frac{1+\zeta_{1} \zeta_{2}}{1+\zeta_{1}^{*} \zeta_{2}}\right)
\end{aligned}
$$

one finds that the output state is again a squeezed vacuum $S\left(z^{\prime \prime}\right) \mid 0,0><$ $0,0 \mid S^{\dagger}\left(z^{\prime \prime}\right)$, and the squeezing parameter $r^{\prime \prime}$ is related to the squeezing parameter $r^{\prime}$ of the input $(a, b)$ and $r^{\prime \prime}$, that of the second OPA, as follows:

$$
\cosh \left(2 r^{\prime \prime}\right)=\cosh (2 r) \cosh \left(2 r^{\prime}\right)+\sinh (2 r) \sinh \left(2 r^{\prime}\right) \cos \alpha,
$$


where $\alpha=\left|\theta-\theta^{\prime}\right|$. This relation sets a limit on the phase difference $\alpha$ beyond which the squeezing parameter for the output and hence its entanglement is less than that of the input. This limiting value $\alpha_{0}$ of $\alpha$ is given by

$$
\begin{aligned}
\cos \alpha_{0}= & -\operatorname{coth} 2 r \tanh r^{\prime} \text { if } r \geq r^{\prime}, \\
& -\operatorname{coth} 2 r^{\prime} \tanh r \text { if } r \leq r^{\prime} .
\end{aligned}
$$

Thus, in order not to degrade quantum entanglement in the output, the phases $\theta$ and $\theta^{\prime}$ should be chosen such that $0 \leq\left|\theta-\theta^{\prime}\right| \leq \alpha_{0}$.

To conclude, we derive the maximal allowable gain of the phase insensitive amplifier for quantum entanglement to survive. Interestingly enough, the entanglement is found to be more robust if only one partner of the pair is amplified which is in agreement with a recent observation 29]. Further, we give explicit results for the growth of entanglement if phase sensitive amplifier is used. Clearly extension of these ideas to non Gaussian entangled states, like

photon subtracted squeezed states [30] and NOON states [31], is a subject of further study.

Acknowledgements One of us (GSA) has enjoyed interesting conversations on entanglement with R. Boyd, J. H. Eberly, P. Knight, M. Plenio and F. Illuminati. This work was supported by NSF Grant no CCF-0829860.

\section{References}

[1] K. Shimoda, H. Takashi, and C. H. Townes, J. Phys. Soc. Japan 12, 686 (1957).

[2] H. A. Haus and J. A. Mullen, Phys. Rev. 128, 2407 (1962).

[3] C. K. Hong, S. R. Friberg, and L. Mandel, J. Opt. Soc. Am. B2, 494 (1985).

[4] G. S. Agarwal and K. Tara, Phys. Rev. A 47, 3160 (1993).

[5] R. Loudon, Philos. Trans. Roy. Soc. London, A 355, 2313 (1997).

[6] M. Artoni and R. Loudon, Phys. Rev. A 57, 622 (1998).

[7] M. S. Kim, W. Son, V. Buzek, and P. L. Knight, Phys. Rev. A 65, 032323 (2002).

[8] P. Diament and M. C. Teich, IEEE J. Quantum Electron. 28, 1325 (1992).

[9] G. Leuchs and U. L. Andersen, Laser Physics 15, 1 (2005).

[10] V. Josse, M. Sabuncu, N. J. Cerf, G. Leuchs, and U. L. Andersen, Phys. Rev. Lett. 96, 163602 (2006).

[11] R. W. Boyd, G. S. Agarwal, K. W. C. Chan, A. K. Jha, and M. N. O'Sullivan, Opt. Comm. 281, 3732 (2008). 
[12] X.-B. Wang, T. Hiroshima, A. Tomita, and M. Hayashi, Phys. Rep. 448, 1 (2007); S. L. Braunstein and P. van Loock, Rev. Mod. Phys. 77, 531 (2005); G. Adesso and F. Illuminati, J. Phys. A 40, 7821 (2007).

[13] A. Furasawa, J. L. Sorensen, S. L. Braunstein, C. A. Fuchs, H. J. Kimble, and E. S. Polzik, Science 282, 706 (1998); W. P. Bowen, N. Treps, B. C. Buchler, R. Schnabel, and P. K. Lam, Phys. Rev. Lett. 89, 253601 (2002).

[14] R. Simon, Phys. Rev. Lett. 84, 2726 (2000).

[15] L.-M. Duan, G. Giedke, J. I. Cirac, and P. Zoller, Phys. Rev. Lett. 84, $2722(2000)$.

[16] G. Giedke, B. Kraus, M. Lewenstein, and J. I. Cirac, Phys. Rev. Lett. 87, 167904 (2001); R. F. Werner and M. M. Wolf, Phys. Rev. Lett. 86, 3658 (2001).

[17] G. Giedke, L.-M. Duan, J. I. Cirac, and P. Zoller, Quantum Inf. Comp. 1, 79 (2002).

[18] R. Simon, E. C. G. Sudarshan, and N. Mukunda, Phys. Rev. A, 36, 3868 (1987); R. Simon, N. Mukunda, and B. Dutta, Phys. Rev. A 49, 1567 (1994).

[19] M. B. Plenio, Phys. Rev. Lett. 95, 090503 (2005); G. Adesso, A. Serafini, and F. Illuminati, Phys. Rev. A 70, 022318 (2004).

[20] G. Vidal and R. F. Werner, Phys. Rev. A 65, 032314(2002); J. Eisert and M. B. Plenio, J. Mod. Opt. 46, 145(1999); S. Virmani and M. B. Plenio, Phys. Lett. A 268, 31 (2000).

[21] S. Scheel, L. Knöll, T. Opatrná, and D.-G. Welsch, Phys. Rev. A 62, 043803 (2000).

[22] M. Hillery and M. S. Zubairy, Phys. Rev. A 74, 032333 (2006).

[23] S. Scheel and D.-G. Welsch, Phys. Rev. A 64, 063811 (2001).

[24] H. Huang and G. S. Agarwal, Phys. Rev. A 49, 52 (1994).

[25] G. S. Agarwal, Phys. Rev. Lett. 97, 023601 (2006).

[26] J. Zhang, C. Ye, F. Gao, and M. Xiao, Phys. Rev. Lett. 101, 233602 (2008).

[27] H. Chen and J. Zhang, Phys. Rev. A 79, 063826 (2009).

[28] A. M. Perelomov, Generalised Coherent States and their Applications, (Springer, 1986), P. K. Aravind, Phys. Rev. A 42, 4077 (1990).

[29] R. C. Pooser, A. M. Marino, V. Boyer, K. M. Jones, and P. D. Lett, Phys. Rev. Lett. 103, 010501 (2009).

[30] G. Adesso, Phys. Rev. A 79, 022315 (2009).

[31] K. T. Kapale and J. P. Dowling, Phys. Rev. Lett. 99, 053602 (2007). 